\title{
PROTEÇÃO E BIOSSEGURANÇA DOS PROFISSIONAIS DE ENFERMAGEM DA ATENÇÃO BÁSICA NO CONTEXTO DA COVID-19
}

\author{
Rosalina Aratani Sudo \\ ORCID: 0000-0002-2206-6671
}

Idenise Vieira Cavalcante Carvalho"

ORCID: 0000-0003-4326-5773

Osvaldo Peralta Bonetti"'

ORCID: 0000-0002-3509-6351

Adriano de Oliveira'

ORCID: 0000-0003-1393-303X

Maria Aureni de Lavor Miranda' ORCID: 0000-0002-0705-3397

Leila Bernarda Donato Göttems ${ }^{\text {IV }}$ ORCID: 0000-0002-2675-8085

I,IV,V Secretaria de Estado de Saúde do Distrito Federal. Brasília, Distrito Federal, Brasil.

"Câmara dos Deputados. Brasília, Distrito Federal, Brasil.

"I'Fundação Osvaldo Cruz. Brasília, Distrito Federal, Brasil.

V' Universidade Católica de Brasília. Brasília, Distrito Federal, Brasil.

I,III,VVVI Membros do Departamento Científico de Enfermagem na Atenção Básica-ABEn seção DF.

\section{Autor Correspondente: \\ Rosalina Aratani Sudo E-mail: rosalinasudo@gmail.com}

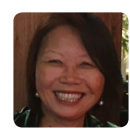

Como citar: Sudo RA, Carvalho IVC, Bonetti OP, Oliveira A, Miranda MAL, Göttems LBD. Proteção e biossegurança dos profissionais de enfermagem da atenção básica no contexto da covid-19. In:Teodósio SSS

Leandro SS (Orgs.). Enfermagem na atenção básica no contexto da COVID-19. 2.ed.rev. Brasília, DF : Editora ABEn, 2020

p. 26-33. (Série Enfermagem e Pandemias, 3). https://doi.org/10.51234/aben.20.e03.c04

\section{INTRODUÇÃO}

A Covid - 19 se alastra rapidamente devido ao alto poder de contágio, quadro agravado pela inexistência de vacinas e medicamentos efetivos. Nesse sentido, prevenir o contágio é a maneira mais eficaz de combatê-la, para tanto, além do necessário isolamento social, o uso dos Equipamentos de Proteção Individual (EPIs) é de fundamental importância para os profissionais de saúde. Por óbvio, em epidemias de doenças altamente infecciosas, como as síndromes respiratórias agudas graves, os profissionais de saúde estão expostos a um risco de contaminação muito maior do que comumente está exposto. (Verbeek et al., 2020).

Em função do alto poder de contágio do novo Coronavírus, transmitido pelo contato físico, pela exposição à aerossóis e gotículas expelidas na saliva, as quais podem persistir por horas ou dias em diversas superfícies, este tema ultrapassou os espaços da saúde e se tornou um ponto de reflexão e disseminação de informação no Brasil e no Mundo (BRASIL, 2020). Devido ao pouco tempo de identificação, ainda temos significativo desconhecimento sobre o comportamento da doença em sua integralidade.

Um ponto ainda bastante polêmico é a incidência de casos de contaminação via pacientes com resultados de exames positivos para a Covid - 19, mas assintomáticos. Embora não saibamos o percentual desta incidência, é preciso considerar que segundo dados da OMS e derivados de pesquisas em curso no mundo, um percentual significativo das transmissões do novo coronavírus pode ocorrer por meio de pessoas assintomáticas, fato que eleva a importância do isolamento social (Kerkhove, 2020). Esse dado destaca a importância dos protocolos clínicos e do uso adequado dos EPIs, por questões de autocuidado, como também, cuidado aos pacientes. Isto porque os profissionais de saúde têm maior capacidade de contaminação do que a população em geral, especialmente aqueles que estão na linha de frente prestando o cuidado 
à população, pela alta exposição a que estão submetidos nos diferentes contextos do cuidado nos serviços de saúde (Sant'Ana et al, 2020).

A escassez de EPIs é um fato vivenciado mundialmente e no Brasil se apresenta com maior criticidade e incidência em função da inexistência de uma política de enfrentamento adequada, por parte da gestão federal da saúde, conforme denúncias cotidianas nos veículos de comunicação. No fim da primeira quinzena do mês de junho, o Brasil já ocupava o lamentável lugar de recordista mundial em número de óbitos a cada 24 horas e de contaminações de profissionais de enfermagem no mundo. Ademais, segundo dados do Conselho Federal de Enfermagem (Cofen), até o dia 19 de junho de 2020, mais de 200 profissionais de enfermagem tinham perdido suas vidas. Tais dados explicitam a importância do desenvolvimento de competências técnicas de forma ágil, do fortalecimento das organizações profissionais e civis para superar o silenciamento frente à cultura autoritária que toma conta da política no país. O momento atual nos traz o imperativo ético de exercício da cidadania entre os profissionais de saúde, além da competência técnica, reivindicando e denunciando a falta de EPI aos trabalhadores da saúde e as precárias condições de trabalho as quais estamos submetidos. (Sant'ana et al, 2020).

Com a pandemia, em função da dramaticidade dos casos graves, os olhares das mídias estão concentrados no debate científico em torno dos serviços hospitalares. Contudo, já existe conhecimento acumulado em relação à efetividade da Atenção Básica à Saúde (ABS) no enfrentamento da pandemia, na medida em que é na rede de unidades básicas de saúde que são realizados os cuidados e as ações de prevenção de doenças e promoção à saúde, nos diferentes territórios onde as pessoas vivem, convivem e se relacionam, em especial aqueles em situação de maior vulnerabilidade sócio sanitária ou de iniquidades em saúde.

Temos uma cultura forjada no conhecimento da infectologia, que nas situações ditas de normalidade sanitária, podemos ter uma postura mais flexível nos serviços de ABS em relação ao uso dos EPIs do que no meio hospitalar. Inclusive, sendo a ABS referenciada na promoção de vínculos efetivos entre profissionais e usuários, em busca de diminuir barreiras e construir relações mais horizontais, muitos profissionais costumeiramente evitam até mesmo o uso do jaleco branco. Contudo, em uma situação pandêmica, é preciso redobrar a importância do uso dos EPIs na ABS. Esta reflexão e as evidências que têm sido compartilhadas, apontam para um longo período de enfrentamento da pandemia, mesmo após o "achatamento da curva" de infecção, o que requer a reinvenção dos processos de trabalho e das práticas profissionais.

Neste cenário, a Associação Brasileira de Enfermagem apresenta algumas contribuições para a maior força de trabalho do SUS, por meio deste E-Book. A sua elaboração foi embasada no conhecimento de Enfermagem acumulado, nos protocolos clínicos nacionais e internacionais disponíveis sobre o tema dos EPIs nos serviços de Atenção Básica.

Na feitura deste material procuramos considerar as múltiplas inserções da enfermagem na atenção básica: a gestão de serviços, das redes e dos territórios; diretamente no cuidado à população; como educadores na promoção da saúde; como docentes e tutores de profissionais em formação. Cada uma destas dimensões da prática da enfermagem na atenção básica está profundamente implicada com o enfrentamento da Covid - 19. O momento requer um nível de conhecimento e acesso à informação efetiva e qualificada que devem ser contextualizados em cada uma das diferentes inserções e espaços nos quais estamos inseridos. Este capítulo tem por objetivo refletir acerca dos requisitos essenciais de biossegurança e contribuir com a prática e o autocuidado dos profissionais de enfermagem que atuam na ABS na atual pandemia.

\section{MUDANÇAS DAS PRÁTICAS DE BIOSSEGURANÇA COMO MEDIDA DE PRECAUÇÃO DAS SÍNDROMES RESPIRATÓRIAS NO CONTEXTO DA ATUAL PANDEMIA}

A ocorrência de uma pandemia por síndrome respiratória aguda com a velocidade de contaminação como a da Covid - 19, exige uma adaptação rápida dos processos de trabalho com pelo menos duas direções: a proteção dos profissionais para que evitem se contaminar e com isto reduzir a capacidade de resposta do 
sistema de saúde; o cuidado direto e coletivo da população que apresenta sintomas da doença e as potencialmente contaminadas e assintomáticas para prevenção de novos casos.

Entre os dispositivos/ferramentas disponíveis neste momento que adquirem grande importância para o processo de trabalho de auto preservação e de proteção da população estão os EPIs, que associado ao treinamento intensivo das equipes, está entre as principais ferramentas de trabalho das equipes da ABS. As ações da enfermagem na ABS devem ser focadas no preparo e proteção dos trabalhadores, na organização do funcionamento dos serviços e na antecipação de suas atividades nas diferentes fases da evolução da resposta à epidemia, o que inclui a previsão e treinamento adequado do uso de EPI (Almeida, 2020).

\section{MEDIDAS DE BIOSSEGURANÇA APLICADAS PELOS PROFISSIONAIS DE ENFERMAGEM DA ABS NO CONTEXTO DA COVID- 19}

As medidas de biossegurança relevantes e indispensáveis sob muitos aspectos no trabalho em saúde, tem elevado seu grau de importância e cuidado no contexto pandêmico hora vivenciado. A biossegurança compreende um conjunto de ações destinadas a prevenir, controlar, mitigar ou eliminar riscos inerentes às atividades que possam interferir ou comprometer a qualidade de vida, a saúde humana e o meio ambiente (BRASIL, 2010). A biossegurança envolve a análise dos riscos a que os profissionais de saúde e de laboratórios estão constantemente expostos em suas atividades e ambientes de trabalho, nos aspectos relacionados aos procedimentos, aos agentes biológicos manipulados, à infraestrutura dos laboratórios ou informacionais, bem como a qualificação das equipes (BRASIL, 2010). Contempla inúmeras normas nacionais e internacionais, as quais não serão abordadas aqui neste documento.

A exposição do trabalhador de saúde a agentes biológicos durante a realização do cuidado direto e indireto pode acontecer por exposição a diferentes fluidos como sangue, urina, fezes e outros. Este risco está presente em vários ambientes de trabalho tais como, unidades básicas de saúde, hospitais, clínicas, ambulatórios, consultórios, em diferentes graus e tempo de exposição a depender do cuidado ali realizado e da quantidade de profissionais presentes. Embora não tenhamos pesquisas que apontem percentuais de contaminação específicos dos trabalhadores da $A B$, a observação dos casos de contaminação de colegas nos dá elementos para afirmar que a contaminação durante o cuidado realizado por enfermeiros na atenção básica é bastante alta. Em função do alto índice, das formas de contágio que perpassam casos sintomáticos e assintomáticos, revelam que a contaminação pode acontecer de múltiplas formas na $A B$, seja no cuidado direto e indireto, no acolhimento dos usuários, nas consultas de enfermagem, durante a testagem que costumeiramente tem sido responsabilidade dos enfermeiros e médicos nas equipes, na visita domiciliar, nas campanhas de vacinação. Enfim, o contágio na pandemia da Covid - 19 pode acontecer em todo momento ou ato que nos coloque em contato com colegas e usuários. Assim, não se restringe aos procedimentos, seja ele invasivo ou não, embora nestes o cuidado deva ser seguido com exatidão. Logo, como toda classe trabalhadora, estamos expostos ao risco desde o deslocamento até as Unidades Básicas de Saúde (UBS), contudo no interior destas a exposição é ainda maior, como também, nas diversas atividades que fazem parte do cuidado de enfermagem nos territórios adscritos.

O uso de EPI nas UBS já era uma recomendação aos profissionais que ali atuam, mas sabe-se que havia dificuldades de adesão e ao mesmo tempo, baixa oferta desses insumos pela concepção equivocada de que o risco que estes serviços oferecem aos profissionais é menor que nos demais ambientes hospitalares e de Unidades de Pronto Atendimento.

Precauções adicionais são exigidas aos profissionais de saúde para se protegerem e impedir transmissão no ambiente de trabalho. Importante que o profissional seja capacitado para o uso correto e retirada do EPI e estabeleça o descarte de acordo com as normas de segurança, pois são considerados materiais potencialmente contaminado (COFEN,2020; ANVISA, 2020.

Em função da escassez de EPI frente à crise gerada pela pandemia, agravada pela carência de investimentos e ausência de políticas governamentais efetivas de proteção ao trabalhador da saúde, a exemplo da necessária 
conversão da indústria, como já referido, têm sido publicadas normativas que indicam a flexibilização do uso da Máscara N95 e afins (BRASIL, ANVISA 2020). Contudo, frente à flexibilização destaca-se: as máscaras são de uso individual, logo não podem ser compartilhadas por trabalhadores em hipótese alguma; caso perceba-se que a máscara está umedecida deverá ser trocada imediatamente (COFEN 2020).

As máscaras de proteção respiratória (por exemplo, N95, PFF2 ou equivalente) e o gorro são necessários apenas para procedimentos que gerem aerossóis (intubação ou aspiração traqueal, ventilação mecânica não invasiva, ressuscitação cardiopulmonar, ventilação manual antes da intubação, coletas de amostras nasotraqueais, broncoscopias, etc.). Importante destacar que a máscara cirúrgica não deve ser sobreposta à máscara N95 ou equivalente, pois além de não garantir proteção de filtração ou de contaminação, também pode levar ao desperdício de mais um EPI, o que pode ser muito prejudicial em um cenário de escassez (COFEN,2020).

O avental não precisa ser necessariamente impermeável, mas o profissional de saúde deve avaliar cada situação e deve utilizar o avental impermeável dependendo do quadro clínico do paciente e a presença de vômitos, diarreia, hipersecreção orotraqueal, sangramento, entre outros. A gramatura indicada para o avental ou capote utilizado em serviços de saúde é de no mínimo $30 \mathrm{~g} / \mathrm{m} 2$ e, no caso de ser impermeável, a gramatura mínima deve ser de $50 \mathrm{~g} / \mathrm{m} 2$.

As luvas de procedimentos não cirúrgicos devem ser utilizadas, no contexto da epidemia da Covid - 19 para precaução de contato. Quando o procedimento a ser realizado no paciente exigir técnica asséptica, devem ser utilizadas luvas estéreis de procedimento cirúrgico.

\section{Requisitos essenciais de biossegurança voltados aos profissionais de enfermagem da ABS na atual pandemia}

As recomendações para o uso dos EPIs em uma UBS também são diferentes a depender dos espaços físicos onde são realizados os cuidados: áreas com trânsito de paciente (pátios e corredores, entre outros), áreas administrativas, farmácias, recepção, sala de espera, sala de acolhimento, sala de isolamento temporário, consultórios, salas para atendimentos ou procedimentos, consultórios odontológicos, salas de imunização, ambulância e outros transportes eletivos de pacientes e domicílio.

Quadro I - Recomendação do uso de EPIs

\begin{tabular}{|c|c|c|c|}
\hline Tipo de Cenário & Público-Alvo & Tipo de Atividade & Tipo de EPI \\
\hline $\begin{array}{l}\text { Áreas } \\
\text { administrativas }\end{array}$ & $\begin{array}{l}\text { Todos os } \\
\text { funcionários }\end{array}$ & Qualquer atividade & Máscara cirúrgica e distância mínima de 1 metro. \\
\hline \multirow{4}{*}{$\begin{array}{l}\text { Área de triagem } \\
\text { externa }\end{array}$} & $\begin{array}{l}\text { Profissionais } \\
\text { da saúde }\end{array}$ & $\begin{array}{l}\text { Triagem de sintomáticos } \\
\text { respiratórios na entrada da } \\
\text { Unidade. Sem contato direto. }\end{array}$ & Máscara cirúrgica e distância mínima de 1 metro. \\
\hline & $\begin{array}{l}\text { Profissionais } \\
\text { da saúde }\end{array}$ & $\begin{array}{l}\text { Acolhimento, classificação de risco, } \\
\text { triagem de enfermagem. }\end{array}$ & $\begin{array}{l}\text { Distância mínima de } 1 \text { metro (quando possível), } \\
\text { máscara cirúrgica, avental impermeável (se risco } \\
\text { de contato com secreção), luvas de procedimento, } \\
\text { protetor ocular ou de face. }\end{array}$ \\
\hline & $\begin{array}{l}\text { Funcionários } \\
\text { ou } \\
\text { Profissionais } \\
\text { da saúde }\end{array}$ & $\begin{array}{l}\text { Provendo orientações, } \\
\text { procedimentos, assistência de } \\
\text { transporte do paciente para uma } \\
\text { instalação de saúde }\end{array}$ & $\begin{array}{l}\text { Máscara cirúrgica, capote, luvas, proteção ocular, } \\
\text { gorro. }\end{array}$ \\
\hline & $\begin{array}{l}\text { Profissionais } \\
\text { da saúde }\end{array}$ & Atividades assistenciais & $\begin{array}{l}\text { Máscara cirúrgica*, capote, luvas, proteção ocular, } \\
\text { sapatos fechados. } \\
\text { *N95 para procedimentos com aerossóis }\end{array}$ \\
\hline
\end{tabular}


Continuação do Quadro 1

\begin{tabular}{|c|c|c|c|}
\hline \multirow{3}{*}{$\begin{array}{l}\text { Consultórios, } \\
\text { sala para } \\
\text { atendimentos ou } \\
\text { procedimentos }\end{array}$} & $\begin{array}{l}\text { Profissionais } \\
\text { de saúde }\end{array}$ & $\begin{array}{l}\text { Exame físico de pacientes com } \\
\text { sintomas gripais. }\end{array}$ & $\begin{array}{l}\text { Máscara cirúrgica, capote ou avental impermeável } \\
\text { (se risco de contato e secreções: luvas de } \\
\text { procedimento, proteção ocular ou de face, gorro. }\end{array}$ \\
\hline & $\begin{array}{l}\text { Profissionais } \\
\text { de saúde }\end{array}$ & $\begin{array}{l}\text { Exame físico de pacientes sem } \\
\text { sintomas gripais. }\end{array}$ & Máscara cirúrgica. \\
\hline & $\begin{array}{l}\text { Profissionais } \\
\text { de saúde }\end{array}$ & $\begin{array}{l}\text { Realização de procedimentos } \\
\text { produtores de aerossóis. }\end{array}$ & $\begin{array}{l}\text { Máscara N95, N99, PFF2 ou PFF3, N99 ou N100, avental } \\
\text { impermeável (se risco de contato com secreção); luvas } \\
\text { de procedimento, proteção ocular ou de face, gorro. }\end{array}$ \\
\hline $\begin{array}{l}\text { Sala de } \\
\text { Imunização }\end{array}$ & $\begin{array}{l}\text { Profissionais } \\
\text { de saúde }\end{array}$ & $\begin{array}{l}\text { Administração de } \\
\text { imunobiológicos. }\end{array}$ & $\begin{array}{l}\text { Máscara cirúrgica, avental, proteção ocular ou de } \\
\text { face. }\end{array}$ \\
\hline $\begin{array}{l}\text { Todos os } \\
\text { ambientes }\end{array}$ & $\begin{array}{l}\text { Profissionais e } \\
\text { Pacientes. }\end{array}$ & Qualquer outra atividade. & $\begin{array}{l}\text { Distância de pelo menos } 1 \text { metro e uso de máscara } \\
\text { cirúrgica (profissionais de saúde) ou tecido } \\
\text { (pacientes). Realizar higienização adequada e } \\
\text { frequente das mãos com produto alcoólico a } 70 \% \\
\text { ou com água e sabonete líquido. }\end{array}$ \\
\hline Comunidade & $\begin{array}{l}\text { Profissionais } \\
\text { de saúde }\end{array}$ & $\begin{array}{l}\text { Assistência direta ao paciente com } \\
\text { sintomas gripais. }\end{array}$ & $\begin{array}{l}\text { Máscara cirúrgica, avental impermeável (se } \\
\text { possibilidade de contato com secreções), luvas de } \\
\text { procedimento, proteção ocular ou de face. }\end{array}$ \\
\hline
\end{tabular}

Fonte: OMS, 2020). Nota Técnica 04/2020 ANVISA

De acordo com ANVISA (2020) todo o material, equipamentos e produtos utilizados na assistência ao paciente com infecção suspeita ou confirmada pelo SARSCoV-2 devem ser submetidos a limpeza e desinfecção ou esterilização de forma a prevenir a possibilidade de contaminação de pele, mucosas e roupas ou a transferência de microrganismos para outros pacientes, profissionais ou ambientes.

Ainda de acordo com a ANVISA (2020), os serviços de saúde devem capacitar todos os profissionais de saúde e de apoio no enfrentamento da Covid -19 e certificar-se do uso dos EPIs adequados antes de cuidar de um caso suspeito ou confirmado de infecção pelo novo coronavírus.

\section{RISCOS ASSOCIADOS A ESCASSEZ E A UTILIZAÇÃO INADEQUADA DE EPI'S/EPC'S E DEMAIS MEDIDAS DE PROTEÇÃO INDIVIDUAL E OU COLETIVA.}

A falta de EPI tem sido um grande problema e quiçá seja uma das explicações dos índices de acometimentos da doença nos profissionais de enfermagem. Frente a esse grave problema, instituições, imprensa, movimentos da saúde pública estão em mobilização nacional e internacional para garantir o abastecimento e a produção e disponibilização adequadas aos profissionais de saúde.

Esta não é uma preocupação somente do Brasil, mas de relevância global. Uma revisão sistemática abrangente que incluiu 36 estudos primários qualitativos, com representatividade de praticamente todos os continentes, aponta dentre as principais preocupações dos profissionais de saúde no enfrentamento às epidemias, provocadas por doenças respiratórias, as questões relacionadas à infraestrutura adequada e à disponibilidade adequada de EPIs. Aponta ainda para a necessidade de planejar o provimento de tais recursos em conformidade com a análise do crescimento do fenômeno epidemiológico (Houghton et al, 2020).

A OMS não recomenda a flexibilização do uso dos EPIs, e sim a racionalização adequada, conforme a atividade realizada pelo profissional de saúde, a partir de estratégias que possam otimizar a disponibilidade de EPIs. (FIOCRUZ, 2020).

Neste contexto, o espaço cotidiano dos serviços é de fundamental importância a ação proativa no planejamento da disponibilização adequada de equipamento de proteção individual e coletiva e o uso racional a fim de evitar a falta, agindo preventivamente. Caso detecte-se que a probabilidade de desabastecimento é alta, deve-se intensificar o diálogo e a reivindicação junto à gestão. Contudo, caso nos deparemos com a falta de EPIs em nossos serviços precisamos estar atentos aos canais de denúncia e proteção. 
Dentre estes espaços destacamos: COFEN, ABEn, Sindicatos da Categoria e da Saúde, Ministério Público, bem como, Conselhos de Saúde e veículos de comunicação e os canais com o legislativo. A central de trabaIhadores da Saúde e Central Única dos Trabalhadores (CNTSS/CUT) lançaram uma campanha "Eu sou o SUS" com foco na valorização e no respeito aos trabalhadores da saúde, a qual também contempla um canal de denúncia e escuta dos trabalhadores da saúde.

Do ponto de vista ético, não custa lembrar que é direito do profissional exercer a Enfermagem com liberdade, segurança técnica, científica e ambiental e com autonomia, conforme definido no Código de Ética dos Profissionais de Enfermagem (COFEN, 2017). Desta forma, o profissional de enfermagem deve avaliar cada situação da assistência de enfermagem, de modo a prestar assistência segura ao paciente e para si mesmo.

De acordo com o Art. 17 da Resolução RDC 63/2011, que dispõe sobre os Requisitos de Boas Práticas de Funcionamento para os Serviços de Saúde, o serviço de saúde deve prover infraestrutura física, recursos humanos, equipamentos, insumos e materiais necessários à operacionalização do serviço de acordo com a demanda, modalidade de assistência prestada e a legislação vigente.

O profissional, ao constatar a ausência de EPI adequado ao atendimento que realiza (verificar quais EPIs são recomendados para cada setor e tipo de atendimento específico conforme Nota Técnica GVIMS/GGTES/ Anvisa - 04/2020), deve procurar os gestores responsáveis pelo seu serviço de saúde para comunicar o fato e solicitar o reabastecimento.

Todavia, recomenda-se que antes de tomar qualquer decisão de negativa da continuidade da assistência, certifique-se de que realmente há desabastecimento dos EPIs. Persistindo o problema do desabastecimento, denuncie junto ao Conselho Regional de Enfermagem do seu estado e demais órgãos de controle.

O profissional poderá suspender suas atividades individuais, quando o local de trabalho não oferecer condições seguras para o exercício profissional e houver risco para sua integridade física, desde que, não seja uma situação de urgência, emergência, epidemia, desastre e catástrofe. Desta forma, o profissional deverá formalizar imediatamente sua decisão por escrito e/ou por meio de correio eletrônico à instituição e ao COREN.

O uso de EPI requer atenção especial tanto no momento da paramentação quanto da desparamentação. O quadro a seguir, demonstra a sequência correta destas duas técnicas de proteção individual:

Quadro 2 - Protocolo de paramentação e desparamentação.

\begin{tabular}{|l|l|}
\hline PARAMENTAÇÃO & DESPARAMENTAÇÃO \\
\hline $1^{\circ}$ Avental ou capote & $1^{\circ}$ Luvas \\
\hline $2^{\circ}$ Máscara cirúrgica OU Máscara de proteção respiratória* & $2^{\circ}$ Avental ou capote \\
\hline $3^{\circ}$ Óculos ou protetor facial & $3^{\circ}$ Gorro ou touca* \\
\hline $4^{\circ}$ Gorro ou touca* & $4^{\circ}$ Óculos ou protetor facial* \\
\hline $5^{\circ}$ Luvas & $5^{\circ}$ Máscara cirúrgica OU de proteção respiratória* \\
\hline${ }^{*}$ No caso de procedimentos geradores de aerossóis. & $\begin{array}{l}\text { OBS: Exceto pela máscara, remova o EPI ainda no quarto, próximo } \\
\text { à saída, ou na antessala. Remova a máscara somente após deixar o } \\
\text { quarto do paciente e fechar a porta. }\end{array}$ \\
\hline
\end{tabular}

Fonte: COFEN, 2020

\section{FATORES QUE INFLUENCIAM A ADESÃO DOS PROFISSIONAIS DE SAÚDE NO USO DE EPI NA PREVENÇÃO DE DOENÇAS INFECCIOSAS RESPIRATÓRIAS E ESTRATÉGIAS DE MELHORIA DA ADESÃO}

Seja pela necessidade de provocar uma sensibilização em cada leitor que é um trabalhador ou pela perspectiva da liderança e papel educacional do profissional enfermeiro, no âmbito da equipe de enfermagem, destacamos a importância de entender as causas da baixa adesão ou adesão seletiva ao uso de EPI, sobretudo 
em ambientes não hospitalares, como é o caso dos serviços da ABS. Neste tópico propõe-se ainda, identificar o que alguns estudos apontam como elementos inerentes ao imaginário dos trabalhadores, e que podem facilitar o trabalho de sensibilização.

Quadro 3 - Fatores contributivos para a adesão dos profissionais de saúde no uso de EPI

\begin{tabular}{|l|l|}
\hline Fatores que dificultam a adesão & Fatores que favorecem a adesão \\
\hline $\begin{array}{l}\text { Diferentes percepções sobre o risco biológico de contágio entre os } \\
\text { profissionais da equipe }\end{array}$ & $\begin{array}{l}\text { Medo de infectar a si mesmos, suas famílias ou por se } \\
\text { sentirem responsáveis pelo contágio de seus pacientes }\end{array}$ \\
\hline $\begin{array}{l}\text { Falta de treinamento adequado sobre o uso dos EPls e demais } \\
\text { estratégias de enfrentamento }\end{array}$ & $\begin{array}{l}\text { Existência de cultura organizacional do serviço favorável a } \\
\text { práticas de segurança do paciente e comunicação efetiva }\end{array}$ \\
\hline $\begin{array}{l}\text { Diretrizes de difícil compreensão seja pelo método de transmissão, seja } \\
\text { pela linguagem ambígua e linguagem pouco clara e pouco objetiva }\end{array}$ & $\begin{array}{l}\text { Nível de apoio da equipe de gerentes percebido pela } \\
\text { equipe de trabalho. }\end{array}$ \\
\hline $\begin{array}{l}\text { Contradições entre as diretrizes locais com as nacionais e } \\
\text { internacionais e mudanças constantes }\end{array}$ & $\begin{array}{l}\text { Decisões compartilhadas em relação ao planejamento e } \\
\text { organização do processo de trabalho da equipe }\end{array}$ \\
\hline $\begin{array}{l}\text { Condições de trabalho e realidade dos serviços desfavoráveis a } \\
\text { prática das diretrizes de enfrentamento da pandemia. }\end{array}$ & $\begin{array}{l}\text { Realização de treinamento com o uso de metodologias } \\
\text { ativas e participativas de ensino aprendizagem }\end{array}$ \\
\hline $\begin{array}{l}\text { Aumento da carga de trabalho e fadiga devido aos procedimentos de } \\
\text { paramentação constantes e a limpeza adicional requerida no ambiente. }\end{array}$ & $\begin{array}{l}\text { Fornecimento de material educativo tais como cartilhas e } \\
\text { livretos }\end{array}$ \\
\hline Desconforto com o uso dos EPls, sobretudo máscaras. & Existência de programas de aprendizagem em serviço. \\
\hline $\begin{array}{l}\text { Sentimento de distanciamento e isolamento em relação aos } \\
\text { pacientes com risco de fortalecer estigmas }\end{array}$ & \\
\hline
\end{tabular}

Fonte: elaborado a partir de Almeida et al (2020), Houghton et al, 2020, Luong et al, 2016.

\section{CONSIDERAÇÕES FINAIS}

Esta publicação demonstra a preocupação da ABEn com utilização dos EPIs, conforme os protocolos e portarias, nos espaços de trabalho da ABS, uma vez que é elevada a probabilidade de um profissional ser infectado ao atender pacientes com Covid - 19 ou manusear amostras biológicas desses pacientes.

Para tanto, fez-se necessário a devida capacitação de todos os profissionais que estão à frente das atividades assistenciais, educacionais e gerenciais, além dos aspectos relacionados ao gerenciamento e manuseio de materiais e resíduos.

Os empregadores deverão intensificar esforços para garantir que cientistas, médicos, enfermeiros, socorristas, laboratoristas e demais profissionais que trabalham nas linhas de frente da pandemia da Covid - 19 recebam, além do equipamento de proteção adequado, as informações necessárias e adequadas para a realização de um trabalho seguro para os pacientes e profissionais.

A organização do processo de trabalho, buscando a separação de pacientes tanto infectados e não infectados por Covid - 19, quanto sintomáticos e assintomáticos, deve caminhar junto com a disponibilidade e uso adequado dos EPIs. Esta nova situação demanda repensar o processo de trabalho das equipes a fim de evitar aglomerações e buscar ao máximo o distanciamento social necessário entre os membros da equipe, embora saiba-se das dificuldades de infraestrutura nos serviços de atenção básica.

\section{REFERÊNCIAS}

1. Verbeek JH, Rajamaki B, ljaz S, Sauni R, Toomey E, Blackwood B, Tikka C, Ruotsalainen JH, Kilinc Balci FS. Personal protective equipment for preventing highly infectious diseases due to exposure to contaminated body fluids in healthcare staff. Cochrane Database of Systematic Reviews 2020, Issue 5. Art. No.: CD011621. DOI: 10.1002/14651858.CD011621.pub5.

2. BRASIL. Ministério da Saúde. Recomendações de proteção aos trabalhadores dos serviços de saúde no atendimento de Covid - 19 e outras síndromes gripais. https://portalarquivos.saude.gov.br/images/pdf/2020/April/16/01-recomendacoes-deprotecao.pdf. 35pag. 
3. - Kerkhove, MV - Pronunciamento sobre transmissão da COVID - 1919 por pacientes assinstomáticos - realizado pela responsável técnica pelo time de combate à Covid - 19-19 da Organização Mundial da Saúde (OMS) Encontrado In: https://agenciabrasil.ebc.com.br/saude/noticia/2020-06/oms-esclarece-que-assintomaticos-podem-transmitir-Covid - 19.

4. Sant'Ana G, Imoto AM, Amorim FF, Taminato M, Peccin MS, Santana LA, Gottems LBD et al. Infecção e óbitos de profissionais de saúde por COVID - 19: revisão sistemática. Acta Paul Enferm. No prelo 2020.

5. Almeida Ildeberto Muniz de. Proteção da saúde em tempos de COVID-19 e respostas à pandemia. Rev. bras. saúde ocup. [Internet]. 2020 [cited 2020 July 06]: 45: e17. Available from: http://www.scielo.br/scielo.php?script=sci_arttext\&pid=S030376572020000101500\&lng=enEpubJune10,2020. https://doi.org/10.1590/scielopreprints.140.

6. Brasil. Ministério da Saúde. Biossegurança em saúde : prioridades e estratégias de ação / Ministério da Saúde, Organização Pan-Americana da Saúde. - Brasília : Ministério da Saúde, 2010.

7. BRASIL. Agência Nacional de Vigilância Sanitária (ANVISA). Nota Técnica (NT) nº 04/2020, sobre as orientações para serviços de saúde: medidas de prevenção e controle que devem ser adotadas durante a assistência aos casos suspeitos ou confirmados de infecção pelo novo coronavírus (SARS-Cov-2). 3a Edição, de 31 de março de 2020.

8. Conselho Federal de Enfermagem (COFEN). Covid - 19 Orientações sobre a Colocação e Retirada dos Equipamentos de Proteção Individual (EPIs). Brasília. 2020. 18pg.

9. Organização Mundial da Saúde (OMS). Tipos Recomendados de Equipamentos de Proteção Individual no Contexto do COVID - 19, de Acordo com o Tipo de Ambiente, Pessoa Alvo e Tipo de Atividade. Disponível in: http://www.abennacional. org.br/site/wp-content/uploads/2020/03/Tabela_Traduzida_EP_OMS.pdf. 2020.

10. Fundação Osvaldo Cruz (FIOCRUZ). Recomendações para a organização da Atenção Primária à Saúde no SUS no enfrentamento da Covid - 19. Série Linha de Cuidado Covid - 19 -19 na Rede de Atenção à Saúde. Maio, 2020. 12pg.

11. Houghton C, Meskell P, Delaney H, Smalle M, Glenton C, Cabine A, Chan XHS, Devane D, Biesty LM. Barreiras e facilitadores à adesão dos profissionais de saúde às diretrizes de prevenção e controle de infecções (IPC) para doenças infecciosas respiratórias: uma rápida síntese qualitativa de evidências. Banco de Dados Cochrane de Revisões Sistemáticas 2020, Edição 4. Art. No .: CD013582. DOI: 10.1002 / 14651858.CD013582.

12. Luong Thanh BY, Laopaiboon M, Koh D, Sakunkoo P, Moe H. Behavioural interventions to promote workers' use of respiratory protective equipment. Cochrane Database of Systematic Reviews 2016, Issue 12. Art. No.: CD010157. DOI: 10.1002/14651858.CD010157.pub2.

13. Ministério da Saúde. Secretaria de Atenção Primária à Saúde (SAPS). Protocolo de manejo clínico do novo coronavírus (Covid - 19) na Atenção Primária à Saúde (Versão 8). Brasília: Ministério da Saúde, 2020. 